\title{
Sevoflurane Effect on Cognitive Function and the Expression of Oxidative Stress Response Proteins in Elderly Patients undergoing Radical Surgery for Lung Cancer
}

\author{
Yang Qin, Jinping Ni, Li Kang, Zhidong Zhong, Liren Wang and Shuzhou Yin
}

\begin{abstract}
Objective: To investigate the effects of sustained inhalation of sevoflurane on cognitive function and the expression of oxidative stress response proteins such as NADPH oxidase subunits NOX2 and NOX4 in elderly patients undergoing radical surgery for lung cancer.

Study Design: An experimental study.

Place and Duration of Study: Department of Anesthesiology, Suzhou Kowloon Hospital, Shanghai Jiao Tong University School of Medicine, China, from February 2016 to October 2017.

Methodology: Elderly patients who underwent radical surgery for lung cancer were divided into the sevoflurane group and the propofol group, with 52 cases in each group. Sustained inhalation of sevoflurane and propofol was administered to maintain anesthesia in the respective groups. Cognitive function and lung function parameters were compared between the two groups. Serum S100 $\beta$ levels and expression of NOX2 and NOX4 proteins in peripheral blood mononuclear cells of the two groups were determined.

Results: At 24 hours after surgery, the lung function indices of the sevoflurane group such as FEV1, FVC and VC were higher than those of the propofol group $(p<0.001, p=0.008$ and $p=0.002$, respectively). At the end of the surgery and at 24 hours after surgery, the MMSE scores of the sevoflurane group were higher than the propofol group (all $p<0.001$ ). S100 $\beta$ levels were lower than the propofol group $(p=0.003$ and $p<0.001$, respectively). Levels of NADPH oxidase subunits NOX2 and NOX4 proteins in peripheral blood mononuclear cells of the sevoflurane group were lower than the propofol group ( $p=0.033, p<0.001, p<0.001$ and $p<0.001$, respectively).

Conclusion: Compared with intravenous anesthesia with propofol, general anesthesia with sevoflurane inhalation has little effect on the short-term cognitive function in elderly patients undergoing radical surgery for lung cancer, and can effectively improve lung function. The mechanism may be related to the reduction of the expression of NOX2 and NOX4 proteins.
\end{abstract}

Key Words: Sevoflurane, Inhalation anesthesia, Elderly patients, Radical resection of lung cancer, Cognitive function, Oxidative stress.

\section{INTRODUCTION}

Cognitive disorders often occur in elderly patients after surgery. The mechanism of postoperative cognitive disorders is relatively complex, involving anesthetic stimulation, surgical trauma and other factors. ${ }^{1}$ Once cognitive disorders occur, the patient will have symptoms such as memory loss and speech disorders, which obviously prolong the patient's hospital stays and seriously affects the patient's prognosis.

At present, radical surgery is the main treatment for elderly lung cancer patients. However, surgical stress and perioperative anesthesia may have an important impact on the cognitive function of elderly lung cancer

Department of Anesthesiology, Suzhou Kowloon Hospital,

Shanghai Jiao Tong University School of Medicine, Suzhou, 215028, China

Correspondence: Shuzhou Yin, Department of Anesthesiology, Suzhou Kowloon Hospital, Shanghai Jiao Tong University School of Medicine, Suzhou, 215028, China

E-mail:wj4ba8bc@sina.com

Received: April 06, 2018; Accepted: September 02, 2018 patients. ${ }^{2}$ For this reason, choosing an appropriate anesthesia programme is particularly important for reducing postoperative cognitive disorders in elderly lung cancer patients. Oxidative stress is a pathological condition that occurs when the redox state of the body cells is out of balance. The mechanism may be related to the involvement and mediation of many proteins such as NADPH oxidase subunits NOX2 and NOX4. ${ }^{3}$ Research has suggested that oxidative stress is one contributing factor to acute lung injury, induced by onelung ventilation. 4

Sevoflurane inhalation anesthesia is an important method of surgical anesthesia. ${ }^{5}$ But the impact of sevoflurane on the cognitive function and the expression of oxidative stress proteins in elderly patients undergoing radical surgery for lung cancer remains unclear.

The objective of this study was to investigate the impacts of sustained inhalation of sevoflurane on the cognitive function, expression of NADPH oxidase subunits NOX2, NOX4 proteins, and lung functionsin in elderly patients undergoing radical surgery for lung cancer, in order to provide a valuable reference for the use of clinical anesthetics in such patients. 


\section{METHODOLOGY}

This study was done in the Department of Anesthesiology, Suzhou Kowloon Hospital, Shanghai Jiao Tong University School of Medicine, China, from February 2016 to October 2017. A total of 104 elderly patients, who underwent radical surgery for lung cancer, were selected. Inclusion criteria were patients who met lung cancer diagnostic criteria and underwent radical surgery for lung cancer, age $\geq 60$, without contraindications to surgery and anesthesia, gave informed consent, and had $\geq 9$ years of education in China. Exclusion criteria were patients with cardio-cerebrovascular diseases, abnormal lung function, cognitive disorders; or a history of drug dependence. The study was approved by the Hospital Ethical and Research Committee, and the patients all signed the Informed Consents. The patients were divided into the sevoflurane group and the propofol group by the random number table method, 52 cases in each group.

Conventional visits were given to all patients before surgery. Venous pathways were established after they entered the operating room. Blood pressure, ECG and other indicators were routinely monitored. Sufentanil $0.5 \mu \mathrm{g} / \mathrm{kg}$, etomidate $0.3 \mathrm{mg} / \mathrm{kg}$ and atracurium $0.5 \mathrm{mg} / \mathrm{kg}$ were given in both groups intravenously to induce anesthesia. Two groups of patients underwent general anesthesia with intubation and one-lung ventilation on their unaffected side. During surgery, the venous targetcontrolled infusion of propofol was given to patients in the propofol group to maintain anesthesia. The sustained inhalation of sevoflurane was given to patients in the sevoflurane group to maintain anesthesia, and the bispectral index (BIS) was controlled within a range of 40 to 60 . During surgery, intravenous sufentanil and atracurium were intermittently administered to maintain analgesia and relax muscles. During skin closure, sevoflurane inhalation was stopped in the sevoflurane group and intravenous propofol was stopped in the propofol group. Patient-controlled intravenous analgesia with $0.8 \mathrm{mg} / \mathrm{kg}$ oxycodone hydrochloride injection was given to all patients two days after surgery. Tracheal extubation was performed when the train-of-four
(TOF) stimulation ratios of the two groups were greater than 0.9 .

The indicators were observed and tested before surgery, at the end of the surgery, and at 24 hours after surgery. A Chinese version of the Mini-Mental State Examination (MMSE) was used to evaluate and compare the cognitive function of the two groups of patients. The MMSE scale score range is $0-30$, of which $27-30$ is considered normal, $21-26$ is mild cognitive dysfunction, $10-20$ is moderate cognitive dysfunction, and $0-9$ is severe cognitive dysfunction. MMSE scores were conducted under supervision of a single expert neuroscientist having more than five years' experience.

The enzyme-linked immunosorbent assay (ELISA) was used to determine and compare the levels of $S 100 \beta$ serum in the two groups. Patients' venous blood was collected. Mononuclear cells were isolated by using the Ficoll lymphocyte separating solution. The Western blotting was used to determine and compare the expression levels of NADPH oxidase subunits NOX2 and NOX4 in peripheral blood mononuclear cells. A gel imager was used to measure the optical density and a quantitative analysis was performed with $\beta$-actin as an internal reference. At the same time, lung function parameters such as forced expiratory volume in one second (FEV1), forced vital capacity (FVC), and vital capacity (VC) were compared between the two groups of patients at 24 hours after surgery.

SPSS 21.0 was used for data analysis. Measurement data were expressed as mean \pm standard deviation. The independent samples t-test was used for the comparison between the two groups. A difference with a p-value less than 0.05 was considered statistically significant.

\section{RESULTS}

One hundred and four patients included 60 males $(57.69 \%)$ and 44 females (42.31\%), aged 60 to 77 years, mean age being $67.63 \pm 2.52$ years. At 24 hours after surgery, the lung function indices of the sevoflurane group such as FEV1, FVC, and VC were higher than those of the propofol group $(p<0.001, p=0.008$ and $p=0.002$, respectively, Table I). Before surgery, there

Table I: Changes of lung function in the two groups at 24 hours after surgery.

\begin{tabular}{|c|c|c|c|c|c|c|c|}
\hline \multirow[t]{2}{*}{ Groups } & \multirow[t]{2}{*}{$\mathrm{n}$} & \multicolumn{2}{|c|}{ FEV1(L) } & \multicolumn{2}{|c|}{$\mathrm{FVC}(\mathrm{L})$} & \multicolumn{2}{|c|}{$\mathrm{VC}(\mathrm{L})$} \\
\hline & & Mean $\pm S D$ & $\mathrm{p}$-value & Mean $\pm S D$ & p-value & Mean \pm SD & $\mathrm{p}$-value \\
\hline Propofol group & 52 & $1.28 \pm 0.27$ & $<0.001$ & $1.89 \pm 0.44$ & 0.008 & $2.61 \pm 0.38$ & 0.002 \\
\hline Sevoflurane group & 52 & $1.56 \pm 0.41$ & & $2.17 \pm 0.59$ & & $2.92 \pm 0.57$ & \\
\hline
\end{tabular}

Table II: MMSE scores and S100 $\beta$ levels of the two groups.

\begin{tabular}{|c|c|c|c|c|c|c|c|c|}
\hline \multirow[t]{2}{*}{ Index } & \multirow[t]{2}{*}{ Groups } & \multirow[t]{2}{*}{$\mathrm{n}$} & \multicolumn{2}{|c|}{ Before surgery } & \multicolumn{2}{|c|}{ At the end of the surgery } & \multicolumn{2}{|c|}{ At $24 \mathrm{~h}$ after surgery } \\
\hline & & & Mean \pm SD & $p$-value & Mean \pm SD & $\mathrm{p}$-value & Mean \pm SD & p-value \\
\hline \multirow[t]{2}{*}{ MMSE (score) } & Propofol group & 52 & $28.76 \pm 0.97$ & 0.573 & $23.02 \pm 0.53$ & $<0.001$ & $27.54 \pm 0.89$ & $<0.001$ \\
\hline & Sevoflurane group & 52 & $28.65 \pm 0.99$ & & $24.98 \pm 0.45$ & & $28.87 \pm 0.54$ & \\
\hline \multirow[t]{2}{*}{$\mathrm{S} 100 \beta(\mathrm{g} / \mathrm{L})$} & Propofol group & 52 & $0.109 \pm 0.004$ & 0.502 & $0.167 \pm 0.027$ & 0.003 & $0.145 \pm 0.018$ & $<0.001$ \\
\hline & Sevoflurane group & 52 & $0.108 \pm 0.010$ & & $0.154 \pm 0.015$ & & $0.126 \pm 0.025$ & \\
\hline
\end{tabular}


was no significant difference in MMSE scores and $S 100 \beta$ levels between the two groups $(p=0.573$ and $p=0.502$, respectively). At the end of the surgery and at 24 hours after surgery, the MMSE scores of the sevoflurane group were higher than the propofol group (all $p<0.001$ ). The $S 100 \beta$ levels were lower than the propofol group $(p=0.003$ and $p<0.001$, respectively, Table II).

Before the surgery, the levels of NADPH oxidase subunits NOX2 and NOX4 proteins in the peripheral blood mononuclear cells of the two groups were not statistically different $(p=0.204$ and $p=0.136$, respectively). At the end of the surgery, the relative expression level of NOX2 protein in the peripheral blood mononuclear cells of the sevoflurane group was $0.55 \pm 0.15$, lower than $0.62 \pm 0.18$ of the propofol group $(p=0.033)$. The relative expression level of NOX4 protein was $0.38 \pm 0.08$, also lower than $0.49 \pm 0.09$ of the propofol group $(p<0.001)$. At 24 hours after surgery, the relative expression level of NOX2 protein in the peripheral blood mononuclear cells of the sevoflurane group was $0.61 \pm 0.05$, lower than $0.74 \pm 0.08$ of the propofol group $(p<0.001)$. The relative expression level of NOX4 protein was $0.42 \pm 0.05$, also lower than $0.56 \pm 0.13$ of the propofol group $(p<0.001)$. Western blotting results of NOX2 and NOX4 proteins in the sevoflurane group are shown in Figure 1.

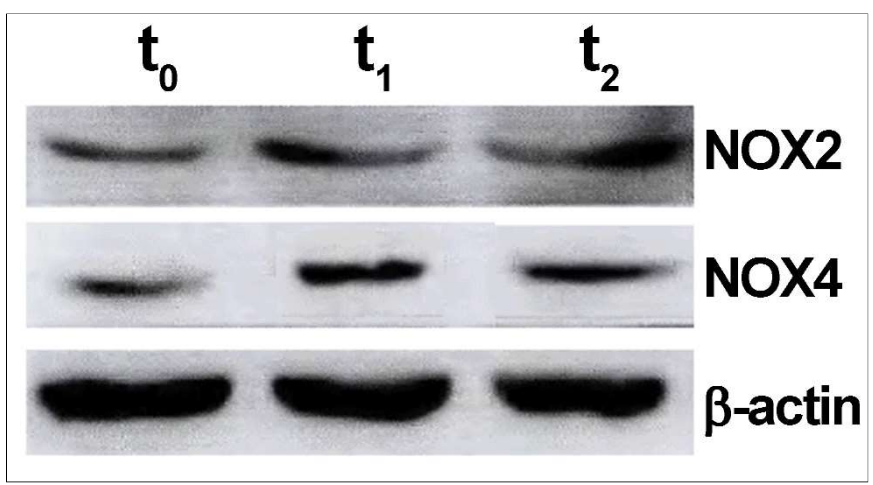

Figure 1: Western blotting results of NOX2 and NOX4 proteins in the sevoflurane group.

\section{DISCUSSION}

The mechanism of postoperative cognitive disorders is still unclear. Recent studies have shown that patients' age, anesthetics, surgical trauma, postoperative infection, etc. are the main factors inducing postoperative cognitive disorders. $6-8$ Among them, anesthetics form the most complex factor. On the one hand, anesthetics play their roles by acting on the central nervous system; on the other hand, they may cause long-term changes in the human nervous system or neurons. ${ }^{9,10}$

Propofol is a relatively common intravenous anesthetic. Studies have confirmed that propofol can play a protective effect on the brain neurons and delay neuronal death. ${ }^{11-13}$ Sevoflurane is a new type of inhaled anesthetic which makes patients wake up faster after surgery. Sevoflurane has a good protective effect on the brain and can reduce the occurrence of brain injury.14-16 Studies have suggested that sevoflurane can reduce the incidence of postoperative cognitive disorders in elderly patients undergoing thoracic surgery. ${ }^{17}$ Some studies have also concluded that both propofol and sevoflurane can affect the cognitive function of elderly patients undergoing orthopaedic surgery, and the latter has the most serious impacts. ${ }^{18}$ The authors found that the MMSE scores in the sevoflurane group were higher than the propofol group both at the end of the surgery and at 24 hours after surgery.

Relevant data showed that $S 100 \beta$ levels were closely related to neurocognitive disorders and an increased level of $S 100 \beta$ could reflect severer postoperative cognitive disorders. ${ }^{19}$ The results of this study showed that the $S 100 \beta$ levels in the sevoflurane group were significantly lower than the propofol group at the end of the surgery and at 24 hours after surgery. It suggested that patients undergoing anesthesia with sevoflurane may suffer a lower risk of postoperative cognitive disorders than those using propofol. A possible reason for this is that the radical surgery for lung cancer can easily induce cerebral hypoxia. Compared with propofol, sevoflurane can better inhibit pulmonary vasoconstriction and reduce pulmonary shunt, and thus better relieve patients' cerebral hypoxia and reduce neuronal damage. 20

Radical surgery for lung cancer is very traumatic to patients, easy to bring intense oxidative stress to patients. Oxidative stress is also an important trigger of lung injury.21,22 The authors found that at 24 hours after surgery, the lung function indices of the sevoflurane group such as FEV1, FVC, and VC were significantly higher than the propofol group, suggesting that patients undergoing anesthesia with sevoflurane had improved lung function than those using propofol. Related data showed that improved lung function was closely related to the regulation of oxidative stress. ${ }^{23} \mathrm{NADPH}$ oxidases are a group of important regulatory proteins in the process of oxidative stress, playing a very important role in oxygen-radical elimination and antioxidation. 24 The results of this study showed that at the end of the surgery and at 24 hours after surgery, the expression levels of NADPH oxidase subunits NOX2 and NOX4 in the peripheral blood mononuclear cells of the sevoflurane group were significantly lower than the propofol group. It suggested that the sevoflurane-based anesthesia could effectively improve the lung function of elderly patients undergoing radical surgery for lung cancer. The mechanism may be related to the regulation of oxidative stress by NADPH oxidase subunits NOX2 and NOX4.

The main limitation and the shortcoming of this study is the fact that the sample size is relatively small. Therefore, further studies should have a larger sample 
and expand the selected area in order to further improve the research.

\section{CONCLUSION}

Compared with propofol-based intravenous anesthesia, general anesthesia based on sevoflurane inhalation has less impact on the short-term cognitive function of elderly patients undergoing radical surgery for lung cancer, and can effectively improve lung function. The mechanism may be related to the reduced expression of NOX2 and NOX4 proteins.

\section{REFERENCES}

1. Cottrell JE, Hartung J. Developmental disability in the young and postoperative cognitive dysfunction in the elderly after anesthesia and surgery: Do data justify changing clinical Practice? Mt Sinai J Med 2012; 79:75-94.

2. Aurini L, White PF. Anesthesia for the elderly outpatient. Curr Opin Anaesthesiol 2014; 27:563-75.

3. Dexter F, Wachtel RE. Strategies for net cost reductions with the expanded role and expertise of anesthesiologists in the perioperative surgical home. Anesth Analg 2014; 118:1062-71.

4. Ronchi CF, Ferreira AL, Campos FJ, Kurokawa CS, Carpi MF, Moraes MA, et al. Interactive effects of mechanical ventilation, inhaled nitric oxide and oxidative stress in acute lung injury. Respir Physiol Neurobiol 2014; 190:118-23.

5. Mohaghegh T, Yazdi B, Norouzi A, Fateh S, Modir H, Mohammadbeigi A. Effect of intravenous anesthesia with propofol versus isoflurane inhalation anesthesia in postoperative pain of inguinal herniotomy: a randomized clinical trial. Med Gas Res 2017; 7:86-92.

6. Grocott HP. Hyperglycemia and postoperative cognitive dysfunction: another call for better glycemic control? Can $J$ Anaesth 2008; 55:140-5.

7. Chen K, Wei P, Zheng Q, Zhou J, Li J. Neuroprotective effects of intravenous lidocaine on early postoperative cognitive dysfunction in elderly patients following spine surgery. Med Sci Monit 2015; 21:1402-7.

8. Praticò $\mathrm{C}$, Quattrone $\mathrm{D}$, Lucanto $\mathrm{T}$, Amato A, Penna $\mathrm{O}$, Roscitano $\mathrm{C}$, et al. Drugs of anesthesia acting on central cholinergic system may cause post-operative cognitive dysfunction and delirium. Med Hypotheses 2005; 65:972-82.

9. Mandal P K, Saharan S, Penna O, Fodale V. Anesthesia issues in central nervous system disorders. Curr Aging Sci 2016; 9:116-43.

10. Stratmann G. Review article: neurotoxicity of anesthetic drugs in the developing brain. Anesth Analg 2011; 113:1170-9.

11. Yamaguchi S, Midorikawa Y, Okuda Y, Kitajima T. Propofol prevents delayed neuronal death following transient forebrain ischemia in gerbils. Can J Anaesth 1999; 46:593-8.

12. Cai J, Hu Y, Li W, Li L, Li S, Zhang M, et al. The neuroprotective effect of propofol against brain ischemia mediated by the glutamatergic signaling pathway in rats. Neurochem Res 2011; 36:1724-31.

13. Luo T, Wu J, Kabadi SV, Sabirzhanov B, Guanciale K, Hanscom M, et al. Propofol limits microglial activation after experimental brain trauma through inhibition of nicotinamide adenine dinucleotide phosphate oxidase. Anesthesiology 2013; 119:1370-88.

14. Chandrasekar A, Heuvel FO, Palmer A, Linkus B, Ludolph AC, Boeckers TM, et al. Acute ethanol administration results in a protective cytokine and neuroinflammatory profile in traumatic brain injury. Int Immunopharmacol 2017; 51:66-75.

15. Sagara Y, Hendler S, Khohreiter S, Gillenwater G, Carlo D, Schubert D, et al. Propofol hemisuccinate protects neuronal cells from oxidative injury. J Neurochem 1999; 73:2524-30.

16. lijima T, Mishima T, Akagawa K, Iwao Y. Neuroprotective effect of propofol on necrosis and apoptosis following oxygenglucose deprivation--relationship between mitochondrial membrane potential and mode of death. Brain Res 2006; 1099:25-32

17. Qiao $Y$, Feng $H$, Zhao $T$, Yan $H$, Zhang $H$, Zhao $X$. Postoperative cognitive dysfunction after inhalational anesthesia in elderly patients undergoing major surgery: the influence of anesthetic technique, cerebral injury and systemic inflammation. BMC Anesthesiol 2015; 15:154.

18. Saricaoglu F, Akinci SB, Oç B, Kanbak M, Akbulut B, Celebioglu $B$. The effect of halothane, isoflurane, sevoflurane and propofol infusion on renal function after coronary artery bypass surgery. Middle East J Anaesthesiol 2006; 18:955-64.

19. He X, Wen LJ, Cui C, Li DR, Teng JF. The significance of S100 $\beta$ protein on postoperative cognitive dysfunction in patients who underwent single valve replacement surgery under general anesthesia. Eur Rev Med Pharmacol Sci 2017; 21:2192-8.

20. Ding J, Fan SD, Li FR. Effect of sevoflurane or propofol anesthesia on intrapulmonary shunting during one-lung ventilation. Jiangsu Med J 2010; 36:166-8.

21. Imai Y, Kuba K, Neely GG, Yaghubian-Malhami R, Perkmann T, van Loo G, et al. Identification of oxidative stress and Toll-like receptor 4 signaling as a key pathway of acute lung injury. Cell 2008; 133:235-49.

22. Kratzer E, Tian Y, Sarich N, Wu T, Meliton A, Leff A, et al. Oxidative stress contributes to lung injury and barrier dysfunction via microtubule destabilization. Am J Respir Cell Mol Biol 2012; 47:688-97.

23. Prado GF, Zanetta DM, Arbex MA, Braga AL, Pereira LA, de Marchi MR, et al. Burnt sugarcane harvesting: particulate matter exposure and the effects on lung function, oxidative stress, and urinary 1-hydroxypyrene. Sci Total Environ 2012; 437:200-8.

24. Ding $\mathrm{H}$, Hashem $\mathrm{M}$, Triggle $\mathrm{C}$. Increased oxidative stress in the streptozotocin-induced diabetic apo E-deficient mouse: changes in expression of NADPH oxidase subunits and eNOS. Eur $J$ Pharmacol 2007; 561:121-8. 